\title{
Collagen Organization in the Secondary Chick Cornea during Development
}

\author{
Andrew J. Quantock, Craig Boote, Veronique Siegler, and Keith M. Meek
}

Purpose. The latter stages of morphogenesis in the embryonic chick cornea are instrumental in the establishment of a properly formed corneal stroma. This study was designed to provide better appreciation of collagen reorganization in the avian corneal stroma during the latter stages of embryogenesis.

Methods. High-angle synchrotron x-ray diffraction patterns were obtained from 47 developing chick corneas daily at developmental days 13 through 18 ( $n=7$ or 8 at each time point) and analyzed to establish collagen molecular spacing and fibril orientation.

Results. Collagen intermolecular x-ray reflections were of approximately constant intensity between days 13 and 15 of development, but thereafter became progressively more intense, suggesting that extra collagen is deposited in embryonic chick corneas after day 16 of development. At all times, the mean collagen intermolecular spacing measured approximately $1.43 \mathrm{~nm}$. X-ray intensity was not uniform around the intermolecular x-ray reflections at earlier time points. Rather, a fourfold symmetry was evident, indicative of an orthogonal array of collagen fibrils. An index of this symmetry was essentially unchanged between developmental days 13 and 15, but thereafter diminished considerably.

Conclusions. The lateral spacing of fibril-forming collagen molecules does not change as the chick cornea develops between days 13 and 18. An orthogonal array of collagen fibrils is present in the corneas of developmental day-13 to -18 chicks, but starting at developmental day 16 , additional collagen is deposited in a less well-oriented manner and thus acts to obscure the overall orthogonality, with implications for the biomechanical strength and shape of the cornea. (Invest Ophthalmol Vis Sci. 2003;44:130-136) DOI:10.1167/iovs.02-0544

$\mathrm{T}$ he mature vertebrate cornea is a remarkable tissue-tough, transparent, and, in terrestrial vertebrates, shaped so as to form the major refractive component of the visual system. In lower vertebrates such as the chicken, the formation of the corneal stroma is the result of a precisely controlled sequence

From the Biophysics Group, Department of Optometry and Vision Sciences, Cardiff University, Cardiff, Wales, United Kingdom.

Presented in part at the annual meeting of the Association for Research in Vision and Ophthalmology, Fort Lauderdale, Florida, May 2002.

Supported by the Medical Research Council (Programme Grant 60001033), the Biotechnology and Biological Sciences Research Council (direct access beamtime award to AJQ), and the TFC Frost Charitable Trust. VS is a doctoral student supported by the TFC Frost Charitable Trust.

Submitted for publication June 5, 2002; accepted July 23, 2002.

Disclosure: A.J. Quantock, None; C. Boote, None; V. Siegler, None; K.M. Meek, None

The publication costs of this article were defrayed in part by page charge payment. This article must therefore be marked "advertisement" in accordance with 18 U.S.C. $\$ 1734$ solely to indicate this fact.

Corresponding author: Andrew J. Quantock, Biophysics Group, Department of Optometry and Vision Sciences, Cardiff University, Redwood Building, King Edward VII Avenue, Cathays Park, Cardiff CF 10 3NB, UK; quantockaj@cf.ac.uk. of developmental events initiated on the third day of incubation by the detachment of the primitive lens from the adjacent surface ectoderm and the subsequent differentiation of this ectodermal layer to form the corneal epithelium (for a review of chick corneal development, see Linsenmayer et al. ${ }^{1}$ ). Soon after, the epithelium synthesizes collagen fibrils, which are secreted into the space between the corneal epithelial basement membrane and the basement membrane of the anterior lens epithelium, so that a loose network of collagen fibrils, 2 to $3 \mu \mathrm{m}$ thick at the end of day 3 , is formed. This primitive, acellular corneal matrix is known as the primary corneal stroma. On the fifth day of development, the primary stroma is rapidly invaded by presumptive corneal fibroblasts that proceed to lay down collagen as the cornea swells and matures into what, in avian species and lower vertebrates, is known as the secondary stroma.

Development of the secondary chick corneal stroma from developmental day 9 to hatching at day 21 is dominated by major structural transformations that govern tissue form and function. The most influential of these include matrix compaction, ${ }^{2-7}$ proteoglycan alterations, ${ }^{8-14}$ and changes in collagenous and noncollagenous extracellular matrix components. ${ }^{15-21}$

Of particular importance when considering structure-function relationships in developing cornea is the matrix of fibrillar collagen and how it is organized. For example, adjustments in the lateral packing of collagen fibrils are believed to be functionally significant, because theories of corneal transparency require some sort of order in the fibrillar array for light transmission. ${ }^{22-26}$ Indeed, early spectrophotometry experiments ${ }^{27}$ indicated that $40 \%$ of incident white light is transmitted through the embryonic chick cornea before and at day 14 of development, with $95 \%$ transmission by day 19 . Thus, some sort of a causal relationship between stromal remodeling during development and the acquisition of corneal transparency seems likely.

Another key structural parameter in corneal development is the orientation of fibrillar collagen. Collagen fibrils that make up the bulk of the stromal matrix in the mature cornea lie in sheets or lamellae in the plane of the cornea. Within each lamella, fibrils lie approximately parallel, but fibrils in adjacent lamellae tend to run at fairly large angles to one another. ${ }^{28}$ The fibrils themselves are composed of long, thin collagen molecules, laterally spaced by approximately $1.6 \mathrm{~nm}$ and axially staggered by defined distances. These collagen molecules (types I and V) tend to run within several degrees of the fibril's axis. Molecular orientation thus approximates fibrillar orientation. In the embryonic chick cornea, we suspect that the orientation of collagen may influence the biomechanical stability, and in turn the shape, of the cornea. To gain better appreciation of the spacing of collagen molecules and the orientation of collagen fibrils within developing chick corneas and any temporal reorganization that might take place, we initiated a high-angle synchrotron $\mathrm{x}$-ray diffraction investigation.

Synchrotron $\mathrm{x}$-ray diffraction is a biophysical technique whereby an intense beam of $x$-rays generated by a synchrotron (a large particle accelerator) is passed through an intact, isolated cornea, ordinarily in a direction at right angles to the 
plane of the cornea, to generate an x-ray diffraction pattern. From this pattern we can determine several details about the cornea's internal structure (for review see Meek and Quantock ${ }^{29}$ ). One feature that can be examined in this manner is the collagen supramolecular structure, and information can be obtained about the mean lateral spacing of fibril-forming collagen molecules, the amount of fibrillar collagen in the cornea, and the orientation of the collagen molecules and fibrils. Data are highly illustrative, because they represent an average throughout the whole region of the cornea through which the $\mathrm{x}$-ray beam passes. Moreover, information is gained from corneas that have not been dehydrated or chemically fixed and thus closely reflect the physiologic situation. Our findings disclosed alterations in the overall collagen orientation, but not lateral intermolecular spacing, in the embryonic chick cornea between days 13 and 18 of development. Predominantly, these are initiated on day 16.

\section{Materials AND Methods}

Fertilized hen eggs were obtained from a commercial hatchery (Poultry First, Worchester, UK) at various stages of development. At this facility, eggs are placed in the incubator daily at around 2 PM and most hatch 21 days and 12 hours later. We collected eggs at noon after 13, 14, 15, 16,17 , and 18 days of incubation and dissected the corneas at the limbus within 3 to 4 hours of collection (at days 13 and 14, this required no incision, because the corneas could simply be grasped at the edge with fine-toothed forceps and pulled away; later time points, however, necessitated circular incisions). At all times the ARVO Statement for the Use of Animals in Ophthalmic and Vision Research was adhered to, as were local rules. Once excised, corneas were immediately plunged into liquid nitrogen-cooled isopentane, wrapped in plastic film to limit evaporation, and stored at $-80^{\circ} \mathrm{C}$ before the synchrotron x-ray diffraction experiments. In all, 47 corneas $(n=7$ or 8 at each developmental day) were used for the current high-angle x-ray diffraction analysis. In their study of freezing in the corneal stroma Fullwood and $\mathrm{Meek}^{30}$ showed that synchrotron x-ray diffraction patterns are essentially unchanged after freezing and thawing, even when the cornea is more highly hydrated than normal.

Specimens were transported frozen to the Synchrotron Radiation Source (SRS) (Daresbury Laboratory, Cheshire, UK) where high-angle synchrotron $\mathrm{x}$-ray diffraction patterns were recorded to provide measurements of the molecular spacing and relative alignments of collagen. A fuller account of the precepts behind this approach as applied to studies of cornea can be found in a recent review. ${ }^{29}$ For the current experiments, corneas were allowed to thaw between two sheets of mylar in an air-tight specimen chamber and were placed in the path of a focused $\mathrm{x}$-ray beam $(\lambda=0.1244 \mathrm{~nm})$, that measured $300 \mu \mathrm{m} \times 300$ $\mu \mathrm{m}$ at the specimen. The $\mathrm{x}$-ray shutter was then opened (for 2 minutes in each case) so that the $x$-ray beam passed through the center of every cornea that was examined in a direction at right angles to the plane of the cornea. X-rays that traveled straight through the specimen were absorbed by a small backstop made of lead placed a few centimeters behind the cornea. Diffracted $\mathrm{x}$-rays were recorded on an $\mathrm{x}$-ray detector located $40 \mathrm{~cm}$ behind the cornea. All $47 \mathrm{x}$-ray diffraction patterns were transferred via the Internet to the home laboratory for analysis on UNIX workstations (Sun Microsystems, Mountain View, CA) and computers with the appropriate Windows NT-based software (Optimas, Bothell, WA) and statistical packages (Excel; Microsoft, Redmond, WA).

A circular $\mathrm{x}$-ray reflection (sometimes lobed) was present on $\mathrm{x}$-ray patterns from all 47 corneas examined. This arose because of the interference of $\mathrm{x}$-rays scattered by collagen molecules within fibrils, and by calibrating the set-up using the sharp $0.305-\mathrm{nm}$ x-ray reflections from powdered calcite, we were able to measure the average lateral separation (Bragg spacing) of the collagen molecules. The relative intensities of these intermolecular reflections provide a measure of the amount of collagen in the volume of the cornea through which the $\mathrm{x}$-ray beam passes (in this case, a volume $300 \mu \mathrm{m} \times 300 \mu \mathrm{m}$ extended through the whole thickness of the cornea). To gauge how much collagen was present, we generated radial distribution plots of $x$-ray intensity in all diffraction patterns, in which the intensity at each radial position was a summation through $360^{\circ}$. A power-law background function, representing x-ray scatter from stromal matrix elements other than fibrillar collagen, was then fitted to and subtracted from the distribution. An index of x-ray intensity, reflective of the amount of collagen, was then calculated for each intermolecular reflection and presented as a ratio of the integrated intensity of the reflection above background to the integrated intensity including background. The amount of aligned collagen as a proportion of total (i.e., aligned plus isotropic) fibrillar collagen was determined from the variation of scattered x-ray intensity measured circumferentially around each intermo-
FigURE 1. High-angle synchrotron $\mathrm{x}$-ray diffraction patterns from chick corneas at developmental days 13 through 18 (D 13-D 18). The central circle is the shadow of a lead backstop used to prevent the main x-ray beam from hitting the $\mathrm{x}$-ray detector. Outside this, regions where scattered $\mathrm{x}$-rays were more intense are indicated in pale yellow. In all cases an $\mathrm{x}$-ray reflection was seen, formed by the interference of $x$-rays scattered by collagen molecules within fibrils. The reflection was lobed at earlier time points. The intensity and position of this reflection allowed the determination of some aspects of the collagen supramolecular structure in the corneal stroma.
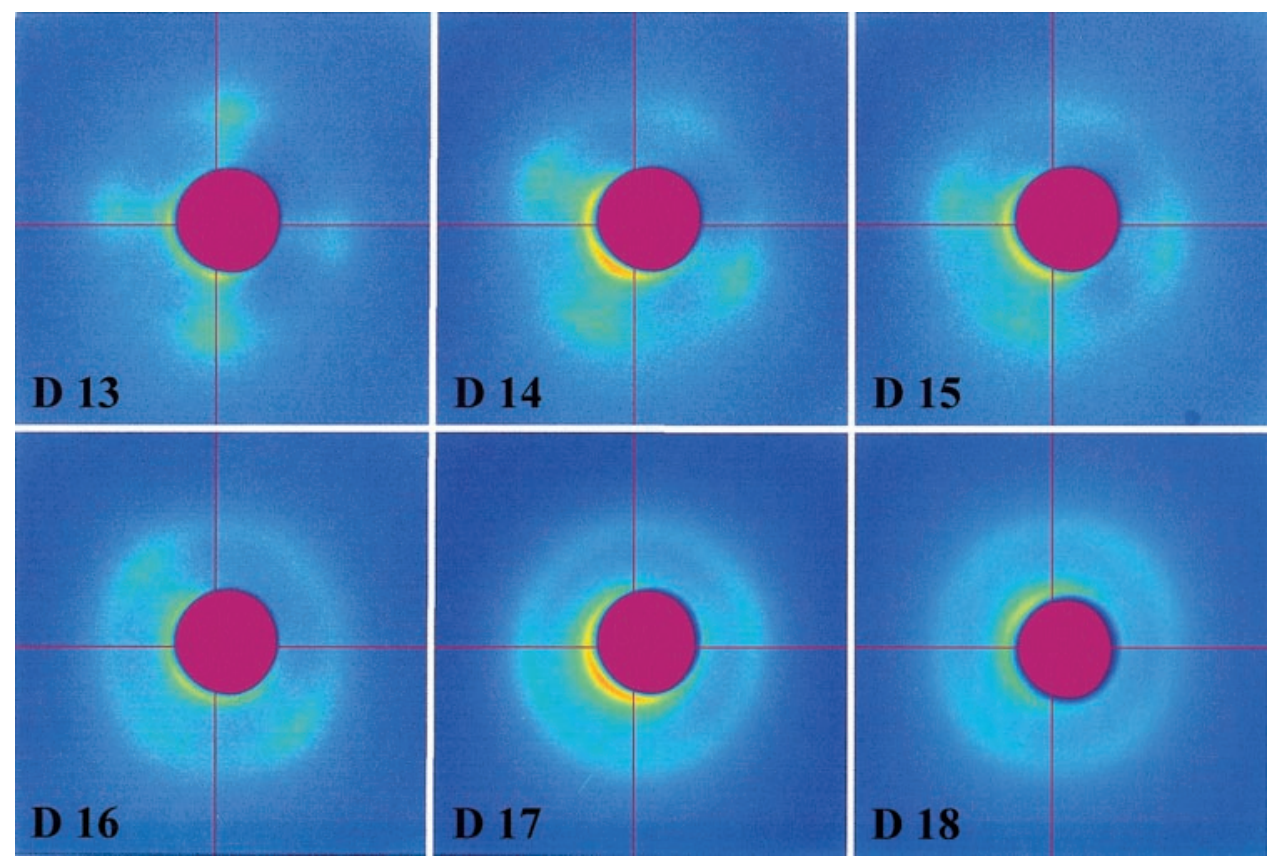


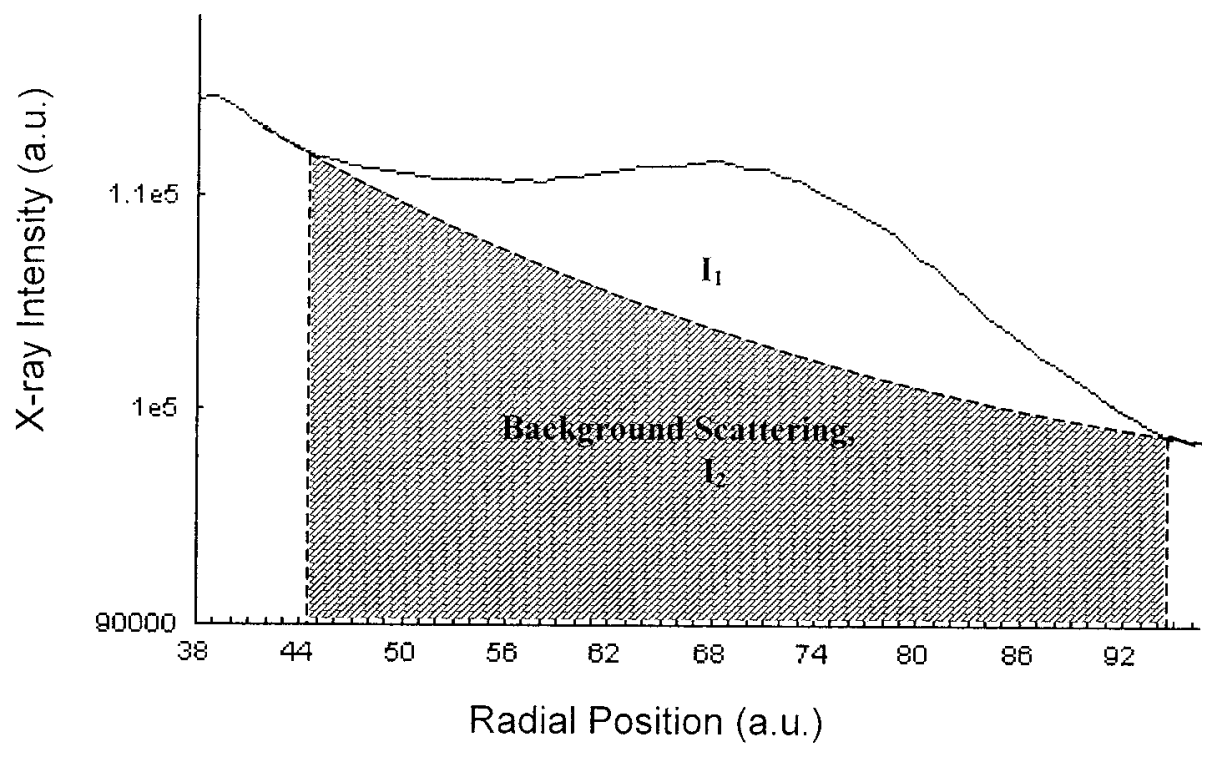

Figure 2. Plot of $\mathrm{x}$-ray intensity against the radial position of the high-angle x-ray diffraction pattern from a day-18 chick cornea over the range that includes the intermolecular reflection. The intensity at each radial position is a summation through $360^{\circ}$. Heightened $x$-ray scattering above background due to the intermolecular reflection is evident and is designated $I_{1}$. The intensity index of each intermolecular $x$-ray reflection is calculated as $I_{1} /\left(I_{1}+I_{2}\right)$. lecular reflection. Typically, fourfold symmetry was evident in the lobed intermolecular reflections, indicative of an orthogonal array of collagen fibrils. A numerical index to represent this orthogonality was calculated as a ratio of $\mathrm{x}$-ray scatter from aligned collagen to $\mathrm{x}$-ray scatter from total collagen.

\section{Results}

High-angle synchrotron x-ray diffraction patterns from all 47 developing chick corneas contained clearly visible $\mathrm{x}$-ray reflections arising from the regularly spaced collagen molecules within fibrils (Fig. 1). Circular, $360^{\circ}$ integrations of the x-ray patterns around their centers, when plotted as a function of the radial distance from the center of the pattern outward, denoted the intermolecular reflections as regions of heightened $\mathrm{x}$-ray intensity (Fig. 2). The intensity peaks were noted to be larger at days 17 and 18 of development than at earlier times, and to investigate this matter in more depth, we generated an index of x-ray intensity for the intermolecular reflec-

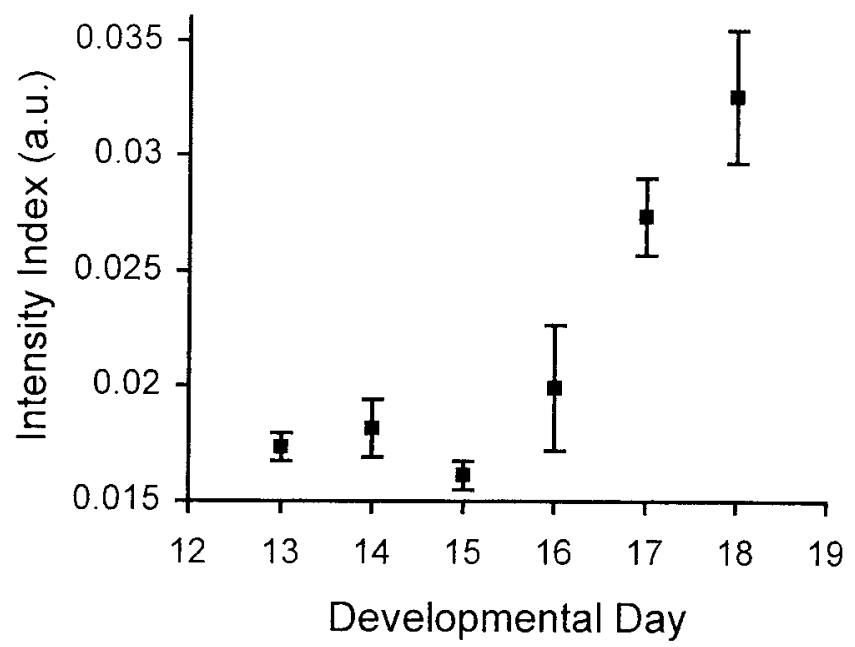

FIGURE 3. Average $( \pm \mathrm{SE})$ intensity indices of the collagen intermolecular reflections for day-13 to -18 developing chick corneas $(n=7$ or 8 at each time point). The trend was for the index to rise progressively after approximately day 16 , indicating the presence of more fibrillar collagen in the cornea at later times. tion for every cornea examined. This index was defined as the ratio of $\mathrm{x}$-ray scattering from regularly arranged collagen molecules above background to total x-ray scattering from all matrix components (including the regularly arranged collagen) over the same radial distance range (Fig. 2). Intermolecular $\mathrm{x}$-ray intensity indices were averaged for all corneas at each developmental day and are presented graphically in Figure 3 (mean $\pm \mathrm{SE}$ ).

Further analysis of the relative positions of the intermolecular reflections enabled us to gain a measure for the mean lateral separation of the fibril-forming collagen molecules. At present, the way in which collagen molecules pack together into fibrils in the cornea is not known, although work in other collagen-containing tissues has suggested that a quasihexagonal or pseudohexagonal mode of packing is likely. ${ }^{31-33}$ Thus, the values obtained and quoted herein are for the average collagen intermolecular Bragg spacing. ${ }^{34}$ Conversion of Bragg spacing to actual intermolecular spacing simply requires multiplication by a packing factor, 1.11 in the case of the pseudohexagonal lattice envisaged by Maroudas et al. ${ }^{33}$ The current data (Table 1) indicate that the mean collagen intermolecular Bragg spacing does not vary appreciably throughout the developmental range studied.

An understanding of how we can elicit information regarding the orientation of collagen fibrils from the collagen intermolecular reflection requires some appreciation of how the X-ray patterns are formed (more detail can be found in Ref. 29). First, because the type I and V collagen molecules that constitute the corneal collagen fibrils tend to lie along the fibrils' axis, we take collagen molecular alignment to reflect collagen fibrillar alignment. An intermolecular x-ray diffraction pattern from a single, isolated lamella in the corneal stroma in which all collagen fibrils run in the same direction would appear as two diffraction maxima located either side of an axis on the x-ray detector that runs in the same direction as the collagen fibrils. Such as situation is depicted in Figure 4, with the diffraction maxima smeared out in a circular manner around the center of the diffraction pattern to indicate a situation in which some fibrils deviate slightly from a perfect parallel arrangement. By extension, two isolated lamellae that lie at right angles to one another would give rise to an x-ray diffraction pattern with four diffraction maxima, equidistant from the center of the x-ray pattern and separated by $90^{\circ}$. In contrast, a stack of lamellae with a totally heterogeneous radial distribution, constructed so 
Table 1. Average Spacing of Collagen Molecules within Fibrils in Day-13 to -18 Developing Chick Corneas

\begin{tabular}{cc}
\hline Developmental Day & $\begin{array}{c}\text { Intermolecular Bragg } \\
\text { Spacing }\end{array}$ \\
\hline $13(n=8)$ & $1.42 \pm 0.03$ \\
$14(n=8)$ & $1.43 \pm 0.03$ \\
$15(n=7)$ & $1.42 \pm 0.02$ \\
$16(n=7)$ & $1.45 \pm 0.02$ \\
$17(n=8)$ & $1.41 \pm 0.02$ \\
$18(n=8)$ & $1.45 \pm 0.01$ \\
\hline
\end{tabular}

Data are $\mathrm{nm}($ mean $\pm \mathrm{SD})$.

that when averaged throughout the whole depth of the cornea no direction in the plane of the cornea contains more fibrils than any other direction, gives rise to a circular intermolecular reflection of uniform intensity around its circumference. The upshot of this is that corneas in which some directions possess proportionally more collagen fibrils than other directions give rise to lobed intermolecular $\mathrm{x}$-ray reflections. Thus, the extent to which intermolecular reflections are lobed allows us to learn about the relative overall orientation of collagen fibrils in the cornea-a line of investigation that has led to the discovery of a preferred superior-inferior/medial-lateral arrangement of collagen fibrils in the center of the mature human cornea ${ }^{35,36}$ and the presence of a circumcorneal annulus of collagen at the limbus, ${ }^{37-39}$ with clear implications for the biomechanical stability of the cornea.

In the current experiments, it was noteworthy that early in development intermolecular x-ray reflections tended to be lobed with fourfold symmetry. Reflections at progressively later developmental days, however, were of a more uniform intensity (Fig. 1). Integrations of $\mathrm{x}$-ray intensity circularly around the $360^{\circ}$ intermolecular reflections were generated to investigate by how much each reflection was lobed. A simple, flat trace would be generated by the integration around a perfectly uniform intermolecular reflection-a trace with four equally spaced peaks by a lobed reflection with fourfold symmetry. Figure 5 shows the circular integrations around the intermolecular reflections from the developmental day-13 and -18 corneas that are presented in Figure 1. From this, it is clear that that the formation of a lobed intermolecular $x$-ray reflection is more conspicuous earlier in development. To quantify

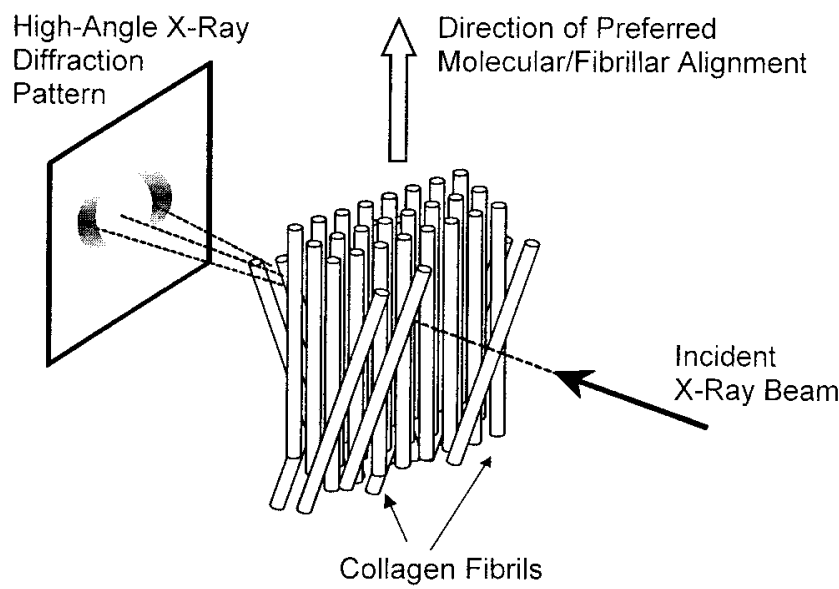

FIGURE 4. Schematic representation of the formation of a high-angle $\mathrm{x}$-ray diffraction pattern from an isolated, single sheet of collagen fibrils, with most fibrils running in the same direction. The reflections shown are due to the collagen molecules within the fibrils that run approximately parallel to the fibrils' axis.

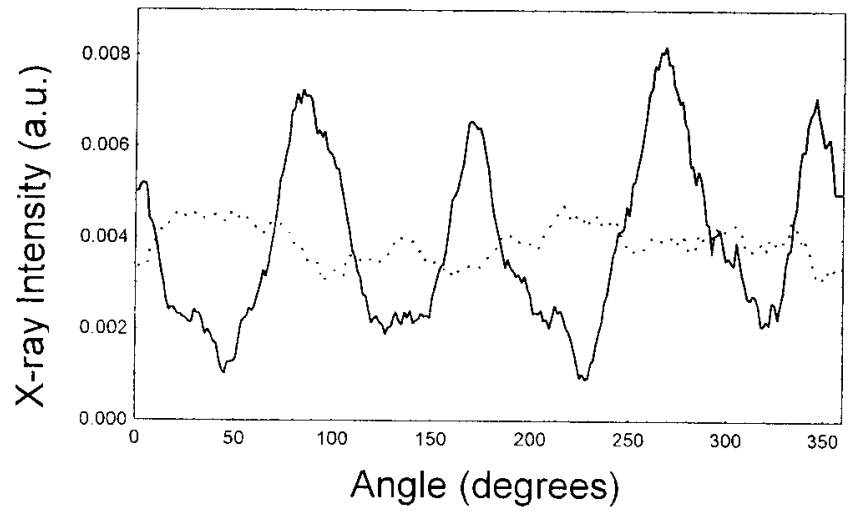

FIGURE 5. Integrated $x$-ray intensity around the intermolecular reflections from the developmental day-13 (solid line) and -18 (dotted line) corneas shown in Figure 1. The four large peaks in the day-13 plot are indicative of a heavily lobed x-ray reflection. The flatter day-18 plot signifies a less lobed intermolecular reflection.

the extent to which each intermolecular reflection was lobed, we calculated a numerical index from the circular integrations from all intermolecular reflections. This was achieved by dividing the area under the peaks but above homogeneous background scatter by the total area under the peaks, including background scatter (Fig. 6). In effect, this is dividing x-ray scatter from orthogonally aligned collagen molecules by the $\mathrm{x}$-ray scatter from all matrix components. As a result, the more lobed the x-ray pattern, the higher its numerical index, and the higher the index, the higher the proportion of orthogonally arranged collagen fibrils in that particular cornea. This analysis revealed that the numerical index describing the extend by which the intermolecular reflections were lobed remained approximately constant between developmental days 13 and 15 , started to decline at day 16 , and diminished further at days 17 and 18 (Fig. 7; mean \pm SD).

\section{Discussion}

All corneas investigated gave rise to a high-angle synchrotron $\mathrm{x}$-ray diffraction pattern with one broad but clear $\mathrm{x}$-ray reflection (Fig. 1). This reflection is formed by the interference of $\mathrm{x}$-rays scattered by the parallel, regularly spaced collagen molecules that make up the stromal collagen fibrils and as a result is commonly known as the intermolecular reflection. Analysis of the relative mean intensities of these reflections indicates that they remain fairly constant between developmental days 13 and 15, but thereafter rise progressively (Fig. 3). Of course, given that the intensity index is a ratio, an increase would occur if the amount of fibrillar collagen remained unchanged throughout development from day 13 to 18 , but the levels of other matrix components diminished. We believe, however, that a more likely cause of the upturn in the x-ray intensity index is the progressive synthesis and formation of collagen molecules into fibrils in embryonic chick corneas that becomes more prominent after day 16 of morphogenesis.

As mentioned, the position of the intermolecular reflection allowed us to calculate the average lateral spacing of types I and $\mathrm{V}$ collagen molecules within the fibrils. This remained essentially unaltered throughout the stages of development studied (Table 1), despite the fact that the secondary chick cornea undergoes considerable dehydration between days 13 and 18 of development. ${ }^{3,4,7}$ This implies that the diameters of the collagen fibrils (unless there are significant changes in the number of molecules present) should not vary. Indeed, this would seem to be the case, given that classic electron micro- 


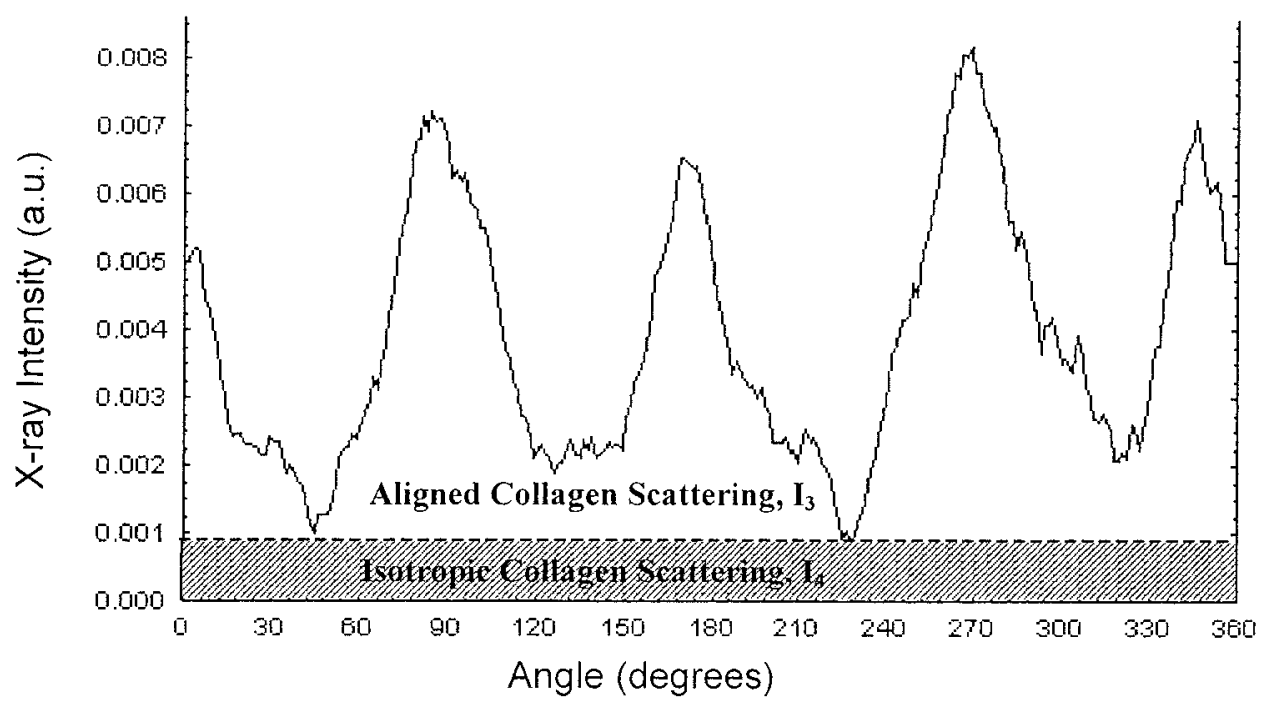

FIGURE 6. Integrated $\mathrm{x}$-ray intensity around the intermolecular reflection (after the subtraction of background $\mathrm{x}$-ray scatter) from a developmental day-13 cornea indicating the contribution to $\mathrm{x}$-ray scatter from preferentially aligned collagen $\left(\mathrm{I}_{3}\right)$ and nonpreferentially aligned collagen $\left(\mathrm{I}_{4}\right)$. The orientation index of each intermolecular $\mathrm{x}$-ray reflection is calculated as $\mathrm{I}_{3} /\left(\mathrm{I}_{3}+\mathrm{I}_{4}\right)$. scopic images of the developing chick cornea disclose collagen fibrils with diameters that remain unchanged during embryogenesis. ${ }^{4,40}$ The finding that water is being removed from the stroma over the day-13 to -18 period with no appreciable compaction of collagen molecules within fibrils is also in line with in vitro biophysical studies of isolated bovine corneas. These divulged that, after dehydration, water is primarily removed from interfibrillar spaces or compartments, and only at levels of tissue hydration much lower than those encountered in the current study (when less than approximately $50 \%$ of the cornea's wet weight is water) is water extracted from within the fibrils themselves, resulting in reduced collagen intermolecular spacing. ${ }^{41,42}$

The fourfold symmetry in the collagen intermolecular reflections became less evident as development proceeded (Fig. 1). This is structurally significant, because it implies that proportionally less collagen fibrils are arranged in an orthogonal array as the chick cornea develops over the period studied (Fig. 7). This is not to say that the orthogonal matrix present at developmental days 13 to 15 is necessarily degraded in any

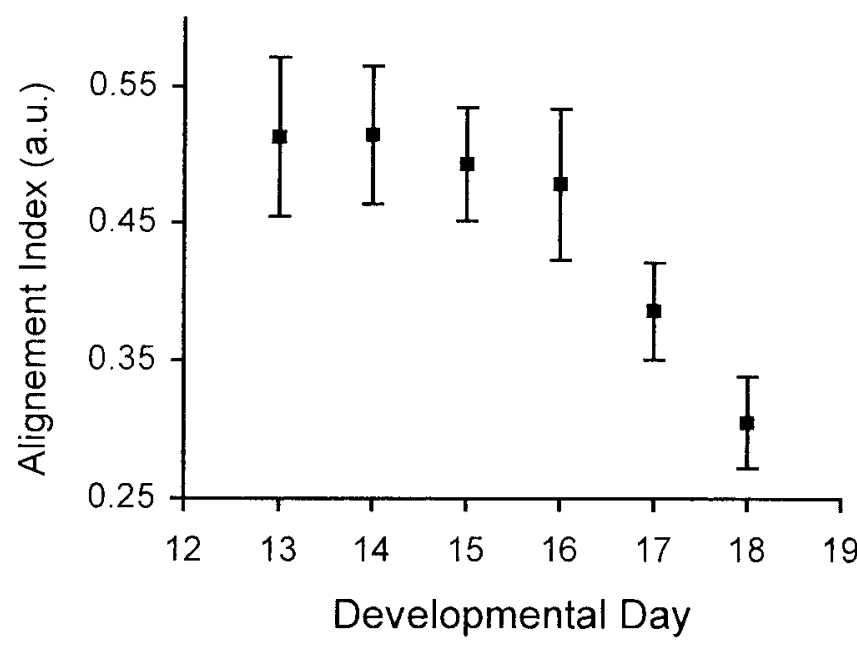

FIGURE 7. Average ( $\pm \mathrm{SE}$ ) numerical indices reflecting the extent to which the intermolecular $x$-ray reflection was lobed at developmental days 13 through 18 ( $n=7$ or 8 at each time point). The index began to decline progressively after approximately day 16, indicating less overall alignment and orthogonality of collagen fibrils as development proceeded. sense. Rather, we believe that the additional regularly arranged collagen that is deposited after day 16 (Fig. 3) is laid down in such a way as to obscure the overall orthogonality of existing collagen fibrils.

How do our findings fit with established knowledge of the developmental dynamics of the embryonic chick cornea? It is well known that the primary stroma up to day 5 of development is deposited by the corneal epithelium and is arranged exclusively in an orthogonal array. ${ }^{4}$ Mesenchyme then invade and quickly begin to synthesize collagen fibrils to form the secondary stroma. These collagen fibrils tend to be deposited in register with the existing lamellae, and thus the orthogonal arrangement is preserved. As a result, the primary corneal stroma has often been viewed as a template or scaffold that dictates the formation of the secondary avian cornea. ${ }^{3,43,44} \mathrm{At}$ the same time as the secondary stroma is being synthesized by presumptive corneal fibroblasts that have invaded it, the epithelium continues to lay down fibrillar collagen. This, of course, is deposited anterior to the invaded stroma and is laid down in lamellae that are rotated a few degrees in relation to the lamella immediately below. As viewed from its endothelial side, the direction of rotation is clockwise when the cornea is traversed from its endothelial to epithelial surfaces. ${ }^{3,44}$ Moreover, the direction of rotation is the same in both eyes, and this lack of axial symmetry around the body midline has led to the suggestion that a self-assembly process based on the inherent "handedness" of fibrillar collagen may be at play. ${ }^{43}$ As development progresses, presumptive fibroblasts invade more of the stroma and continue to lay down collagen in register with existing fibrils, and because deposition by the epithelium of successively rotated layers of collagen is continuing, the newly deposited secondary stromal lamellae are also rotated.

The upshot of this developmental sequence of events is that in both developmental day- $12^{44}$ and -14 corneas $^{43}$ the posterior $50 \mu \mathrm{m}$ of stroma (approximately 25\% stromal thickness at these times) comprises an orthogonal array of collagen fibrils. This is simply because the secondary stromal fibrils in the deepest layers of the cornea were laid down in register with the orthogonal fibrils of the primary stroma before the deposition of the anterior rotated lamellae had begun. Collagen lamellae anterior to this $50-\mu \mathrm{m}$ zone are progressively rotated, and throughout the remaining $150 \mu \mathrm{m}$ of the anterior rotated stroma at day 14 the total net angular displacement is approximately $210^{\circ} .{ }^{43}$ Recent work has shown that the small-angle light scattering and birefringence properties of the mature chick cornea are consistent with this structural model. ${ }^{45}$ 
Our finding of collagen intermolecular $\mathrm{x}$-ray reflections with fourfold symmetry at days 13 to 15 of development can be reconciled with the tissue architecture we have outlined if we remember that the $\mathrm{x}$-ray patterns we obtain are generated by passing the $x$-ray beam through the whole thickness of the cornea. Thus, $x$-ray scatter from all levels of the tissue-that is, anterior rotated, and posterior unrotated-contributes to the pattern at these and other times. If we first consider $x$-ray scatter from the regularly arranged collagen molecules in the anterior rotated stroma, we can reason that if the degree of rotation between successive lamellae is assumed to be approximately equal (and at day 14 of development this was estimated by Trelstad and Coulombre ${ }^{43}$ to be between $2.8^{\circ}$ and $5.6^{\circ}$ per lamellae), a net rotation of $180^{\circ}$, or any multiple thereof, would lead to a fully homogeneous angular distribution of fibrils with no preferred fibrillar orientation and no lobing of the intermolecular reflection. We contend that a net rotation in the region of $210^{\circ}$ that is predicted at day 14 of development ${ }^{43}$ is close enough to $180^{\circ}$ to give rise to an intermolecular reflection with no readily discernible lobes. Of course, because the $\mathrm{x}$-rays pass through the whole cornea, $\mathrm{x}$-ray scatter from regularly spaced collagen molecules in the posterior, unrotated stroma would also contribute to the $\mathrm{x}$-ray diffraction pattern, and at days 13 to 15 this comprises approximately one quarter of the stromal thickness. Because collagen fibrils in this portion of the stroma lie in an orthogonal array, they would be expected to give rise to an intermolecular reflection with fourfold symmetry that would be superimposed on the isotropic scatter from nonpreferentially arranged anterior collagen to form a lobed intermolecular reflection. Indeed, this is what we saw, especially at days 13 to 15 . In this way our x-ray data fit with the established view of corneal matrix ultrastructure during this period of development. $^{3,43,44}$

An important finding of the present study is that $\mathrm{x}$-ray diffraction patterns obtained from corneas between developmental days 16 and 18 differ from patterns obtained at earlier time points, because the intermolecular reflection is more homogeneous (Figs. 1, 7), a finding that, as mentioned, is indicative of proportionally less orthogonal, aligned collagen in the cornea at these later times. Clearly, this situation could be arrived at by the reorganization of collagen fibrils and lamellae in the posterior, unrotated part of the cornea into a less orthogonal array or by continued deposition of fibrillar collagen in such a way as to mask the contribution to the intermolecular reflection of the posterior orthogonal lamellae. We cannot provide any direct evidence to support our contention, but believe that the latter possibility is by far the more likely, especially because changes in the intensity index of the intermolecular reflection (Fig. 3) point to the deposition of increased amounts of collagen after day 16 of development.

Given that the x-ray diffraction patterns analyzed were generated by passing x-rays through the whole thickness of the cornea, our data can shed no light on the possible location in relation to stromal depth of the collagen that we envisage is deposited at and after day 16 of development and that obscures the orthogonality of the fibrillar array in the posterior cornea. Nevertheless, it is interesting to note that Linsenmayer et al. ${ }^{46}$ have documented two layers of stable collagen in the anterior half of the embryonic chick cornea that are well established by day 18 to 19 of embryogenesis and that run uninterrupted from limbus to limbus and appear to associate with the scleral ossicles. They indicate that at developmental days 12 to 14 the stable collagen bands are faint and less apparent in the central cornea than at the periphery. They further conclude that the collagen bands do not develop abruptly, but form slowly over a period of days. It is conceivable that the fibrillar collagen that we contend is deposited in the central cornea at and after day 16 of development is, at least in part, the collagen of the stable bands. Moreover, if by providing a physical link to the scleral bundles and scleral ossicles, the function of the stable bands is to promote corneal stability as has been proposed, ${ }^{46}$ this may require an angular distribution of collagen fibrils that has no preferential alignment. Such an arrangement would act to obscure the orthogonality in the posterior cornea on x-ray diffraction patterns.

\section{Acknowledgments}

The authors thank Rob Keyhoe, Mike McDonald, and Mike Baily for help at the Synchrotron Radiation Source.

\section{References}

1. Linsenmayer TF, Fitch JM, Gordon MK, et al. Development and roles of collagenous matrices in the embryonic avian cornea. Prog Retinal Eye Res. 1998;17:231-265.

2. Smelser GK, Ozanics V. Distribution of radioactive sulfate in the developing eye. Am J Ophthalmol. 1957;44:102-110.

3. Coulombre AJ, Coulombre JL. The development of the structural and optical properties of the cornea. In: Smelser GK, ed. The Structure of the Eye. New York: Academic Press; 1961:405-420.

4. Hay ED, Revel J-P. Fine structure of the developing avian cornea. In: Wolsky A, Chen PS, eds. Monographs in Developmental Biology. Vol. 1. Basel, Switzerland: S. Karger; 1969:1-144.

5. Quantock AJ, Kinoshita S, Capel MC, Schanzlin DJ. A synchrotron $\mathrm{x}$-ray diffraction study of developing chick corneas. Biopbys J. 1998;74:995-998.

6. Hirsch M, Noske W, Prenant G, Renard G. Fine structure of the developing avian corneal stroma as revealed by quick-freeze, deepetch electron microscopy. Exp Eye Res. 1999;69:267-277.

7. Siegler V, Quantock AJ. Two stage compaction of the secondary avian cornea during development. Exp Eye Res. 2002;74:427-431.

8. Anseth A. Glycosaminoglycans in the developing corneal stroma. Exp Eye Res. 1961;1:116-121.

9. Toole BP, Trelstad RL. Hyaluronate production and removal during corneal development in chick. Dev Biol. 1971;26:28-35.

10. Hart GW. Biosynthesis of glycosaminoglycans during corneal development. J Biol Chem. 1976;251:6513-6521.

11. Funderburgh JL, Caterson B, Conrad GW. Keratan sulfate proteoglycan during embryonic development of the chicken cornea. Dev Biol. 1986;116:267-277.

12. Cornuet PK, Blochberger TC, Hassell JR. Molecular polymorphism of lumican during corneal development. Invest Ophthalmol Vis Sci. $1994 ; 35: 870-877$.

13. Cai CX, Gibney E, Gordon MK, Marchant JK, Birk DE, Linsenmayer TF. Characterization and developmental regulation of avian corneal $\beta$-1, 4-galactosyltransferase mRNA. Exp Eye Res. 1996;63:193200.

14. Dunlevy JR, Beales MP, Berryhill BL, Cornuet PK, Hassell JR. Expression of the keratan sulfate proteoglycans lumican, keratocan and osteoglycin/mimecan during chick corneal development. Exp Eye Res. 2000;70:349-362.

15. Birk DE, Fitch JM, Linsenmayer TF. Organisation of collagen types $\mathrm{I}$ and $\mathrm{V}$ in the embryonic chicken cornea. Invest Opbthalmol Vis Sci. 1986;27:1470-1477.

16. Birk DE, Fitch JF, Babiarz JP, Doane KJ, Linsenmayer TF. Collagen fibrillogenesis in vitro: interaction of types I and $\mathrm{V}$ collagen regulates fibril diameter. J Cell Sci. 1990;95:649-657.

17. Linsenmayer TF, Fitch JM, Schmid TM, et al. Monoclonal antibodies against chicken type V collagen: production, specificity, and use for immunocytochemical localization in embryonic cornea and other organs. J Cell Biol. 1983;96:124-132.

18. Linsenmayer TF, Fitch JM, Mayne R. Extracellular matrices in the developing avian eye: type $\mathrm{V}$ collagen in corneal and noncorneal tissues. Invest Ophthalmol Vis Sci. 1984;25:41-47.

19. Linsenmayer TF, Fitch JM, Birk DE. Heterotypic collagen fibrils and stabilising collagens: controlling elements in corneal morphogenesis. Ann NY Acad Sci. 1990;580:143-160.

20. Fitch JM, Birk DE, Linsenmayer C, Linsenmayer TF. Stromal assemblies containing collagen types IV and VI and fibronectin in the developing embryonic avian cornea. Dev Biol. 1991;144:379-391. 
21. Fitch JM, Gordon MK, Gibney EP, Linsenmayer TF. Analysis of transcriptional isoforms of collagens types IX, II, and I in the developing avian cornea by competitive polymerase chain reaction. Dev Dyn. 1995;202:42-53.

22. Maurice DM. The structure and transparency of the cornea. J Pbysiol (Lond). 1957;186:263-286.

23. Hart RW, Farrell RA. Light scattering in the cornea. J Opt Soc Am 1969;59:766-774.

24. Benedek GB. Theory of transparency of the eye. Appl Opt. 1971; 10:459-473.

25. Freund DE, McCally RL, Farrell RA. Direct summation of fields for light scattering by fibrils with applications to normal corneas. $A p p l$ Opt. 1986;25:2739-2746.

26. Farrell RA. Corneal transparency. In: Albert DM, Jacobiec SA, eds. Principles and Practice of Opbthalmology. Philadelphia: WB Saunders; 1994.

27. Coulombre AJ, Coulombre JL. Corneal development. I. Corneal transparency. J Cell Comp Physiol. 1958;51:1-11.

28. Komai Y, Ushiki T. The three-dimensional organization of collagen fibrils in the human cornea and sclera. Invest Opbthalmol Vis Sci. 1991;32:2244-2258

29. Meek KM, Quantock AJ. The use of x-ray scattering techniques to determine corneal ultrastructure. Prog Retinal Eye Res. 2001;20: 95-137.

30. Fullwood NJ, Meek KM. An ultrastructural, time-resolved study of freezing in the corneal stroma. J Mol Biol. 1994;236:749-758.

31. Hulmes DJS, Miller A. Quasi-hexagonal molecular packing in collagen fibrils. Nature. 1979;282:878-880.

32. Fraser RD, MacRae TP, Miller A, Suzuki E. Molecular conformation and packing in collagen fibrils. J Mol Biol. 1983;167:497-521.

33. Maroudas A, Wachtel E, Grushko G, Katz EP, Weinberg P. The effect of osmotic and mechanical pressures on water partitioning in articular cartilage. Biochem Biophys Acta. 1991;1073:285-294.

34. Meek KM, Leonard DW. The ultrastructure of the corneal stroma: a comparative study. Biophys J. 1993;64:273-280.
35. Meek KM, Blamires T, Elliott GF, Gyi T, Nave C. The organisation of collagen fibrils in the human corneal stroma: a synchrotron X-ray diffraction study. Curr Eye Res. 1987;6:841-846.

36. Daxer A, Fratzl P. Collagen fibril orientation in the human corneal stroma and its implications in keratoconus. Invest Ophthalmol Vis Sci. 1997;38:121-129.

37. Newton RH, Meek KM. Circum-corneal annulus of collagen fibrils in the human limbus. Invest Opbthalmol Vis Sci. 1998;39:11251134.

38. Newton RH, Meek KM. The integration of the corneal and limbal fibrils in the human eye. Biopbys J. 1998;75:2508-2512.

39. Meek KM, Newton RH. Organisation of collagen fibrils in the corneal stroma in relation to mechanical properties and surgical practice. J Refract Surg. 1999;15:695-699.

40. Birk DE, Trelstad RL. Extracellular compartments in matrix morphogenesis: collagen fibril, bindle, and lamellar formation by corneal fibroblasts. J Cell Biol. 1984;99:2024-2033.

41. Meek KM, Fullwood NJ, Cooke PH, et al. Synchrotron x-ray diffraction studies of the cornea with implications for stromal hydration. Biopbys J. 1991;60:467-474.

42. Fratzl P, Daxer A. Structural transformation of collagen fibrils in corneal stroma during drying: an x-ray scattering study. Biophys J. 1993;64:1210 - 1214

43. Trelstad RL, Coulombre AJ. Morphogenesis of the collagenous stroma in the chick cornea. J Cell Biol. 1971;50:840-858.

44. Coulombre J, Coulombre A. Corneal development. V. Treatment of five-day-old embryos of domestic fowl with 6-diazo-5-oxy-L-norleucine (DON). Dev Biol. 1975;45:291-303.

45. McCally RL, Farrell RA. Small-angle light scattering and birefringence properties of chick cornea. J Refract Surg. 1999;15:706710

46. Linsenmayer TF, Gibney E, Fitch JM. Embryonic avian cornea contains layers of collagen with greater than average stability. J Cell Biol. 1986;103:1587-1593. 